\title{
Psicoterapia psicoanalítica
}

\section{Psychoanalitical pshycotherapy}

\author{
Juan Diego Lopera Echavarría 1 ORCID \\ 1 Universidad de Antioquia \\ Colombia
}

Fecha correspondencia: Recibido: mayo 2 de 2016. Aceptado: enero 12 de 2017.

Forma de citar: Lopera, J. (2017). Psicoterapia psicoanalítica. Rev. CES Psicol., 10(1), 83-98.

$\underline{\text { Open access }}$

c) Copyright

Licencia creative commons

Etica de publicaciones

Revisión por pares

Gestión por Open Journal System

DOI: http://dx.doi.org/10.21615/

cesp.10.1.6

ISSN: 2011-3080

Sobre los autores:

1. Doctor en Ciencias Sociales,

Magister en Filosofía, Psicólogo

e investigador Departamento

de Psicología, Universidad de

Antioquia

Comparte

\section{Resumen}

El artículo tiene como objetivo analizar una de las prácticas más importantes de la orientación psicoanalítica: la psicoterapia, como posible respuesta, entre otras, a la pregunta por las relaciones entre la psicología y el psicoanálisis. Se presentan algunos antecedentes de la psicoterapia, se plantea una definición de la misma, se exponen dos clases generales para entender los propósitos psicoterapéuticos (ascéticos y sintomales) y se establecen algunas afinidades y divergencias a partir de tres categorías en las que se pueden ubicar diferentes modalidades psicoterapéuticas: a) el conocimiento psicológico y la transmisión de información al consultante; b) la relación terapéutica; y c) la transmisión de una actitud analítica al paciente. El presente manuscrito se propone desde una mirada pluralista e indogmática establecer tanto las afinidades (lo general) en las modalidades psicoterapéuticas como sus diferencias (lo específico). Muestra que la psicoterapia se remonta a la tradición filosófica del cuidado del alma (therapeuein heauton), tanto en un sentido específico (sintomal) como amplio (ascesis subjetiva). Considera que la focalización, más correspondiente con las psicoterapias sintomales, no se opone ni impide un trabajo de revisión exhaustiva de la vida del paciente. Privilegia, de los diversos modos de enfocar el trabajo psicoterapéutico, aquel que se basa en la transmisión de una actitud (analítica) como forma de abordar la existencia; en consecuencia, se distancia de la concepción de la psicología clínica como la aplicación del conocimiento psicológico al ámbito clínico.

Palabras clave: Psicoterapia, Psicoanálisis, Ascético, Sintomal

\section{Abstract}

This paper aims to analyze one of the most important practices of the psychoanalytic orientation: psychotherapy, as a possible answer, among others, to the question about the connections between psychology and psychoanalysis. A background of psychotherapy is presented, and it is set up a definition of its concept. In addition, two main categories are discussed (ascetical and symptomal), in order to understand better the psychotherapeutic purposes. Some similarities and differences are established departing from three categories in which psychotherapeutic approaches are located: 1) the psychological knowledge and the transfer of information to the consultant 2) the therapeutic relationship and 3) the transmission of an analytical attitude to the patient. This paper arose from a pluralistic and non-dogmatic point of view in order to establish both similarities (overaII) and differences (particularities) in psychotherapeutic approaches. It is 
shown how psychotherapy dates back to the philosophical tradition of soul caring (therapeuein heauton), both in a specific sense (symptomal) and an overall sense (subjective ascesis).It is considered that the focus of the symptomal psychotherapies does not prevent an exhaustive revision of the patient's life. This article favors the different approaches for the psychotherapeutic work, that one based on the transmission of an (analytical) attitude as a way to face one's life; in consequence, it takes distance from the conception of clinical psychology as the application of psychological knowledge to the clinical domain.

Keywords: Psychotherapy, Psychoanalysis, Ascetic, Symptomal.

\section{Introducción}

La orientación psicoanalítica constituye un amplio espectro de conceptualizaciones y formas de abordaje sobre lo psíquico. No es una escuela sino más bien una orientación metodológica (Gil, 1995; Lopera, Ramírez, Zuluaga, \& Ortiz, 2010; Ramírez, 2011; Ramírez, et al., 2015), en la que confluyen diferentes propuestas que tuvieron sus orígenes en el psicoanálisis freudiano. Freud diferenció claramente tres aspectos en su descripción del psicoanálisis: el método de investigación de procesos anímicos inconscientes, la terapia fundada en esa indagación y el conjunto de conocimientos que derivan de ese camino (Freud, 1923/1998). Este último aspecto, denominado por Freud como psicología de lo inconsciente o psicología de lo profundo (Freud, 1915/1998, p. 169), ha dado lugar a la diversidad de enfoques psicoanalíticos en la explicación de los fenómenos psíquicos. Unas corrientes conservan los fundamentos del psicoanálisis clásico; otras, a partir de una reformulación radical, proponen una superación definitiva de la teoría freudiana; otras más, ocupan un lugar intermedio y, aunque conservan algunas ideas fundamentales de Freud, elaboran otros conceptos, desarrollan nuevas perspectivas de abordaje psicoterapéutico (Mitchel \& Black, 2004). En tanto estos enfoques proponen una explicación de lo psíquico, son perspectivas psicológicas, lo que nos habla de una orientación psicoanalítica en la psicología, al lado de las orientaciones conductuales, experimentales, cognitivas, existenciales, humanistas, transpersonales (Ramírez, et al., 2015).

Entre las propuestas enmarcadas en la orientación psicoanalítica encontramos convergencias y divergencias. Si pretendiésemos hallar la razón por la que son llamadas psicoanalíticas, y lo hiciéramos a partir del establecimiento de un conjunto de conocimientos o de conceptos básicos que todas deberían tener, correríamos el riesgo de privilegiar unas y subvalorar otras, puesto que cada una hace énfasis distintos: en las relaciones objetales, o en la tensión entre pulsión y defensa, en lo intrapsíquico, entre otros aspectos (Kernberg, 2001; 1993). Por esto, es más apropiado plantear que la razón para que sean psicoanalíticas, pese a sus grandes diferencias, estriba en un enfoque metodológico que, por un lado, considera esencial lo inconsciente en las investigaciones, análisis y reflexiones sobre el alma'; y por el otro, privilegia la subjetividad en el trabajo clínico ${ }^{2}$ individual o grupal, sin desconocer el contexto social y cultural.

Este artículo aporta algunos elementos de reflexión sobre la pregunta por las relaciones entre la psicología y el psicoanálisis, a partir de examinar una de sus derivaciones: la psicoterapia psicoanalítica. Esta pregunta ha sido central en nuestro con-

1 El concepto Alma lo entendemos como cultura encarnada (Lopera, Zuluaga, Manrique, \& Ortiz, 2010) basándonos en las elaboraciones de Michel Foucault, quien la concibe como efecto de las operaciones de la cultura sobre los cuerpos (Foucault, 1981, p. 36). Se trataría del resultado del proceso de sujetación del individuo a la cultura, lo que se conoce como subjetivación.

2 Una manera de conceptualizar la clínica en correspondencia con la orientación psicoanalítica, puede consultarse en Manrique, Lopera, Pérez, Ramírez y Henao (2016) 
Pág 85

Foucault, por su parte, resalta la diversidad de técnicas que, en la antigüedad griega y romana, estaban destinadas al cuidado de sí (Foucault, 1990; 2000), con lo que se destaca la importancia que tuvo el abordaje de la psyché (el alma) desde el punto de vista de su cultivo y ascesis. No obstante, durante la Edad Media, y sobre todo con la creación de las universidades, se fue perdiendo gradualmente la concepción de la filosofía como terapéutica y modo de vida, relevándose por la de un discurso teórico alejado de la vida (Hadot, 2006). tinente y en Europa (Jardim \& Rojas, 2010; Kernberg, 2001; León, s.f.; Santamaría, 2002), así como en nuestro contexto local ${ }^{3}$.

\section{Algunos antecedentes filosóficos}

Desde la antigüedad clásica el hombre se ha ocupado del alma (psyché) con el propósito de construir unos conocimientos sobre la misma (tendencia epistémica), pero también desde la pretensión de cuidarla y cultivarla (tendencia ascética) (Lopera, 2006). El cuidado de sí (epimeleia heautou) (Foucault, 2002, p. 17; Lopera, 2016) es la expresión directa de una terapéutica que pretende una ascesis subjetiva: una transformación radical del ser humano con el propósito de alcanzar la eudaimonía o buen vivir. La imagen que nos transmite Platón de Sócrates, es precisamente la de un filósofo interesado en cuestionar a los demás con el propósito de que prestaran atención a su propia alma, y no a otro tipo de bienes (Platón, 1985, 312c; 1988, 66b). Para Hadot (1998, p. 38) la "misión de Sócrates es pues hacer tomar conciencia a los hombres de su no saber", para que dirijan la mirada hacia sí mismos. Aristóteles (1985, 1102a15) resaltó la phrónesis (moderación, prudencia) como lo más importante en la obra de un filósofo, entendida como una sabiduría práctica que tiende a un modo de vida virtuoso, excelso. Aubenque (1999) muestra el lugar central de la prudencia en la concepción filosófica de Aristóteles, en la que se destaca la forma de vida tendiente a la virtud, mediante una transformación del ser humano.

Según Hadot (1998; 2006), las filosofías antiguas eran fundamentalmente una terapéutica para las angustias del alma, un modo de vida, más que un discurso filosófico. Destaca que, no obstante, las diferencias entre los socráticos, los aristotélicos, los estoicos, los epicúreos, los cínicos y los escépticos, había una afinidad en la importancia concedida a la búsqueda de la serenidad de espíritu, mediante el cuidado de sí, el cultivo del alma (Hadot, 2009, p. 177).

Foucault, por su parte, resalta la diversidad de técnicas que, en la antigüedad griega y romana, estaban destinadas al cuidado de sí (Foucault, 1990; 2000), con lo que se destaca la importancia que tuvo el abordaje de la psyché (el alma) desde el punto de vista de su cultivo y ascesis. No obstante, durante la Edad Media, y sobre todo con la creación de las universidades, se fue perdiendo gradualmente la concepción de la filosofía como terapéutica y modo de vida, relevándose por la de un discurso teórico alejado de la vida (Hadot, 2006). Con el advenimiento de la época moderna y la importancia que revistió el método científico (Brett, 1972; Cassirer, 1932/1994; Descartes, 1637/2008), las reflexiones sobre el alma privilegiaron la vertiente epistémica basadas preferentemente en el método experimental.

La ascesis fue mirada con desconfianza, como asunto que sólo competía a la religión, a la metafísica o a unas prácticas supersticiosas relacionadas con las creencias populares. Se deslindaron de manera radical dos perspectivas: por un lado, las reflexiones (científicas) sobre el alma entendidas como construcción de conocimientos (psicología epistémica) y, por el otro, las experiencias y prácticas tendientes al cuidado del alma (psicología ascética y psicoterapia en sentido amplio). Téngase en cuenta que al hablar de psicología y de psicoterapia en este contexto,

3 En nuestro medio, específicamente en la Universidad de Antioquia (Medellín, Colombia), el programa de psicología fue fundado en 1975 con la intención de brindar una formación psicológica que contara con el psicoanálisis, sin que eso significara la pretensión de formar psicoanalistas en la Universidad (Consejo Directivo Universidad de Antioquia, 1975, 1979; Lopera, 2015). Esa sola propuesta generó una aguda polémica entre quienes creían en ese proyecto y quienes no, llevando a unos debates que retrasaron la aprobación oficial del Departamento de Psicología (Departamento de Psicología Universidad de Antioquia, 1986; ICFES, 1975; 1977; 1982; 1984; Lopera, 2015; López, 1995) y, posteriormente, a la separación de un grupo de profesores que crearon el Departamento de Psicoanálisis en la Universidad, diferente del Departamento de Psicología (Consejo Superior Universidad de Antioquia, 1990; Sección de Servicios del Departamento de Psicología Universidad de Antioquia, 1989). En la actualidad, el Departamento de Psicología cuenta con varios cursos de psicoanálisis y una amplia oferta de cursos socio-humanísticos, algunos de ellos con enfoque psicoanalítico (Departamento de Psicología Universidad de Antioquia, 2008). 
se está haciendo referencia a dos abordajes sobre lo psíquico que atraviesan gran parte de la historia de la filosofía, y no exclusivamente a la psicología autodenominada científica a partir del siglo XIX y comienzos del Xx (Brett, 1972; Lopera, Manrique, Zuluaga, \& Ortiz, 2010).

\section{El psicoanálisis}

El psicoanálisis desde su origen se ubicó en una posición paradójica con relación a las tendencias que primaban en ese momento (finales del siglo XIX y comienzos del $\mathrm{xx}$ ): mientras la psicología estaba interesada en constituirse en una disciplina científica experimental y poca importancia (o nula) concedía a lo terapéutico (Dilthey, 1945; Watson, 1982/1916; Wundt, 1982/1896), Freud estaba interesado en la curación de las neurosis, en el cuidado y tratamiento del alma (Freud, 1890/1998), y se basaba en una modalidad no experimental del método científico: el paradigma indiciario (Ginzburg, 1989), fundamento del método psicoanalítico por él propuesto.

Inmerso en el espíritu científico de la época, Freud consideraba que todo abordaje del alma digno de consideración debía basarse en un proceder rigurosamente científico. Afirmó que "el psicoanálisis es un método de investigación, un instrumento neutral, como lo es, por ejemplo, el cálculo infinitesimal" (Freud, 1927/1998, p. 36). Lo definió como un método de investigación de procesos anímicos inconscientes, una terapia fundada en esa investigación y un conjunto de conocimientos psicológicos ganados por ese camino (Freud, 1923/1998, p. 231). Continuaba con la tradición ascética mediante la aplicación del método científico a un proceso de ascesis subjetiva (Lopera, Ramírez et al., 2010). Esta situación quizá explique la razón por la cual los debates acerca de la cientificidad del psicoanálisis surgieron desde el momento mismo de su origen.

En un comienzo Freud pretendía una curación de los síntomas de las neurosis, pero su trabajo de investigación le mostró la necesidad de ampliar el campo de análisis hacia la infancia del sujeto, privilegiando así esa indagación que conduciría, por añadidura, a la modificación de los síntomas (Freud, 1923/1998, pp. 246-247; 1912/1998). Y de manera más precisa, en lo que corresponde a entender su propuesta psicoterapéutica como un proceso de ascesis subjetiva, dice: "Nosotros, los analistas, nos proponemos como meta un análisis del paciente lo más completo y profundo posible; [...]. Lo que de tal suerte cultivamos es cura de almas en el mejor sentido" (Freud, 1926/1998, p. 240). Este énfasis en lo ascético no significó una subvaloración de los síntomas, pues en algunas circunstancias era necesario un trabajo sobre los síntomas concretos, ya presentes, mediante una terapia sintomal (Freud, 1895/1998, p. 269), con lo que se complementaría el trabajo de ascesis subjetiva.

Muy tempranamente Freud definió psicoterapia como tratamiento (terapia) del alma (psique); en sus palabras:

(...) tratamiento desde el alma -ya sea de perturbaciones anímicas o corporales- con recursos que de manera primaria e inmediata influyen sobre lo anímico del hombre. Un recurso de esa índole es sobre todo la palabra, y las palabras son, en efecto, el instrumento esencial del tratamiento anímico (Freud, 1890/1998, p. 115).

Esta definición es aplicable a la manera como Freud concibió el tratamiento analítico hasta el final de su vida, y que se confirma con la cita en la que se refiere a la cura de almas. 


\section{La psicología freudiana y sus derivaciones}

Freud construyó una serie de conocimientos sobre el psiquismo, su proceso de constitución y lógica de funcionamiento. Estas teorizaciones fueron llamadas psicología de lo inconsciente, psicología profunda (Freud, 1923/1998, p. 247), psicología del ello. En conjunto, consisten en la metapsicología freudiana (Freud, 1915/1998, p. 178), punto de partida para las elaboraciones de sus discípulos y de los autores considerados postfreudianos. Estas construcciones posteriores se conocen como teorías psicodinámicas (o más propiamente psicoanalíticas), fundamento de las distintas vertientes actuales. De esas teorías iniciales de Freud derivan: la orientación filosófica y existencial (Binswanger, 1973); la psicología del yo desarrollada inicialmente por Hartmann (1964); la teoría de las relaciones objetales en varias vertientes: aquella que privilegia el concepto de relación (Mahler, 1972), la que da más importancia al objeto (internalizado), y la que da igual peso al factor pulsional y a la relación de objeto; las psicologías de la identidad y del self; y la perspectiva culturalista, entre otras (Kernberg, 2001).

Wyss (1964) realiza una clasificación por grupos - el grupo británico, el neoyorquino, entre otros- y por temas, clasificación con la que Bleichmar y Leiberman (1997) coinciden parcialmente; Stephen Mitchel y Margaret Black (2004) retoman autores clásicos y analizan las propuestas de los que llaman revisionistas contemporáneos, como Jacques Lacan, Otto Kernberg, Roy Schafer y Hans Loewald; Otto Kernberg (1993; 2001) analiza las divergencias y convergencias en las concepciones sobre la técnica psicoanalítica contemporánea.

Si bien existen confluencias en las múltiples propuestas también existen profundas diferencias, razón por la cual, al denominar psicología psicoanalítica ${ }^{4}$ nos estamos refiriendo a la orientación psicoanalítica en la psicología con sus múltiples vertientes. Otto Kernberg es uno de los autores que más se ha interesado en señalar estas diferencias, sin descuidar las afinidades. En cuanto a las divergencias más importantes señala, en el aspecto técnico, la oposición entre quienes hacen énfasis en una perspectiva constructivista (en especial, en el análisis interpersonal e intersubjetivo), que privilegian la relación que se construye en el aquí y en el ahora entre analista y paciente, por contraste con el análisis llamado estándar, clásico, que considera esencial los aspectos transferenciales derivados del pasado (allá y entonces) (Kernberg, 2001, pp. 204-205). Asimismo, existen diferencias en cuanto a los énfasis teóricos sobre lo que denomina motivación inconsciente: para algunos, la teoría pulsional; para otros las relaciones objetales (en contra del privilegio del factor pulsional, que deja en segundo plano la importancia de la relación de objeto en la estructuración del psiquismo) y, para otros más, "la teoría de la organización estructural ("primera y segunda tópica" en el psicoanálisis francés)" (Kernberg, 2001, p. 206).

Ramírez (2011) propone como elementos centrales y confluyentes entre las diversas corrientes, los siguientes aspectos: la importancia concedida a la escucha de las formaciones de lo inconsciente, la verbalización, y el análisis y manejo de la transferencia; todo ello basado en la aplicación del método psicoanalítico:

\footnotetext{
4 Somos conscientes de las críticas que genera hablar de psicología psicoanalítica. En nuestro medio, por ejemplo (MedellínColombia) ha tomado carrera la idea de que o se trata de psicoanálisis o se trata de psicología, no de una mezcla de las dos (Pérez, 1992; Ramírez, 1994). Sin embargo, entendemos psicología en un sentido amplio, no solamente académico, relacionada con la tradición filosófica: cualquier estudio riguroso y sistemático sobre lo psíquico (alma), que busque articular (logos) mediante razones, argumentos y teorías los conocimientos adquiridos sobre el alma, es psicología. Así, el psicoanálisis es psicología en este sentido amplio; pero en otro sentido se distancia de ella por la importancia que le concede a la ascesis subjetiva. (cfr. Ramírez et al., 2015, en particular, el capítulo seis, titulado El psicoanálisis como ciencia crítico-emancipatoria y como experiencia de ascesis subjetiva). Ahora bien, hablar de psicología psicoanalítica es referir un estudio sobre el alma que tiene en cuenta los presupuestos del método psicoanalítico.
} 
La orientación psicoanalítica en la psicología se basa en la aplicación del método psicoanalítico en la práctica psicológica, incluyendo la investigación o construcción teórica. El método psicoanalítico es el análisis de un discurso mediante la contrastación dialéctica entre el discurso (teoría) y la experiencia (práctica) a partir de la escucha de las formaciones de lo inconsciente, y se sustenta en una apertura hacia el discurso inconsciente que permite su análisis y la posterior intervención del analítico. La orientación psicoanalítica, al estar basada en una actitud, se diferencia de la psicología dinámica, que se sustenta en una teoría particular sobre lo psíquico que es aplicada al trabajo psicológico (2011, p. 72).

Resta señalar, sin pretender profundizar en este aspecto, que no ignoramos la diferencia entre psicología y psicoanálisis, aunque nos interese mostrar sus afinidades. El psicoanálisis es fundamentalmente un método de investigación del alma; de allí que se denomine psyché-análysis, resaltando la perspectiva analítica como procedimiento básico; la psicología, en cambio, es esencialmente un tratado, un conjunto de conocimientos articulados sobre el alma, lo que se destaca en su denominación: psyché-logos. Asimismo, dada esta diferencia, se derivan otras: el psicoanálisis ha privilegiado la experiencia de transformación o ascesis subjetiva, en la que los aspectos singulares e incluso peculiares son los que se tienen en cuenta (Ramírez, 2012a); la psicología, por su parte, en tanto ha buscado constituirse en una ciencia, deja de lado esos aspectos singulares (no generalizables en conjuntos de conocimientos válidos) propios de un proceso de transformación subjetiva. No obstante, psicología y psicoterapia han estado muy relacionados, al punto de que esta última se considera una aplicación de la primera. Conviene, en consecuencia, centrarnos en la psicoterapia propiamente dicha.

\section{La psicoterapia: ascética y sintomal}

La psicoterapia ha sido central en la psicología psicoanalítica, lo que ha llevado a que muchos homologuen, de manera errónea, psicología psicoanalítica con psicoterapia psicoanalítica. La psicoterapia se define como un tratamiento del alma con el propósito de moderar el sufrimiento o de transmitir una actitud ante la existencia. La psicología, incluso en su vertiente ascética, abarca muchos más aspectos, tanto a nivel teórico como práctico, en particular, procura la construcción de un conocimiento sobre lo psíquico: psyché-logos, que literalmente significa tratado, discurso sobre el alma (Lopera, Manrique et al., 2010). En cambio, psyché-therapeuein sería cuidado, cura del alma, en el sentido de therapeuein heauton (cuidado de sí) (Foucault, 2002, p. 110; 1987). Desde esta perspectiva, el tratamiento psicoanalítico sería una modalidad (entre otras) de psicoterapia.

El dispositivo terapéutico freudiano consistió en un artificio metodológico (Ramírez, 1991) fundamentado en cuatro elementos: asociación libre (verbalización), atención flotante (escucha analítica), análisis y manejo de la transferencia (vínculo o relación paciente-analista) e interpretación (conjunto de intervenciones) (Freud, 1912/1998). A partir de esta propuesta diversos psicoanalistas han elaborado otras, modificando tanto el proceder técnico como la psicopatología o caracterología correspondientes con dichas innovaciones. En sentido general, podemos agruparlas en psicoterapias ascéticas y sintomales (Lopera, 2006).

La mayoría de psicoterapias psicoanalíticas dan vital importancia a un trabajo de revisión exhaustiva de la existencia del paciente: un proceso en el que se busca la modificación subjetiva más radical posible, lo que llamamos psicoterapia ascética. Hemos entendido la palabra ascesis no en el sentido cristiano de renuncia y mortifi- 
Pág 89

La tendencia ascética no desconoce la importancia de que los síntomas y problemas específicos puedan resolverse; no obstante, le da más énfasis a ese cambio psíquico profundo, porque lo considera correlativo con un modo de vida más acorde con las posibilidades del paciente. cación, sino en el del despliegue del propio ser, que comprende la puesta en juego de las potencialidades y la elaboración constante de sí, mediante un cuidado de la propia alma (Foucault, 2002; 1993; Lopera, 2016; Lopera, Ramírez et al., 2010; Ramírez et al., 2015). En Freud este propósito es claro. En las propuestas derivadas de Klein, Bion, Anna Freud, Fairbairn, Winnicott, Kohut y otros, se busca que la relación terapéutica pueda crear las condiciones más favorables posibles para que el paciente pueda desplegarse - partiendo, si es del caso, de ese punto en el que su desarrollo fue perturbado- y su auténtica personalidad, o su auténtico self, puedan expresarse naturalmente. El énfasis puesto cada vez más en las relaciones de objeto tempranas (Kernberg, 1993) muestra un trabajo exhaustivo que contempla, no sólo las experiencias presentes de la vida del paciente -el aquí y ahora-, sino también las vivencias más arcaicas de la infancia (Kernberg, 2001). Guntrip, refiriéndose al psicoanálisis en tanto tratamiento, sostiene que en éste se buscan resultados radicales mediante una exploración analítica profunda y cabal (1971, p. 174). Miller (2001) propone una diferencia entre psicoanálisis (puro y aplicado a la psicoterapia) y psicoterapia, señalando que esta última no tiene en cuenta el goce y, en consecuencia, deja intacta la idea de la omnipotencia del otro, incluso la preserva.

La tendencia ascética no desconoce la importancia de que los síntomas y problemas específicos puedan resolverse; no obstante, le da más énfasis a ese cambio psíquico profundo, porque lo considera correlativo con un modo de vida más acorde con las posibilidades del paciente. En ocasiones, esa ascesis lleva a que el paciente reconozca sus limitaciones, capacidades, elecciones y, aunque no necesariamente esto hace la vida más confortable, sí más acorde con su ser, más armónica con su subjetividad.

En este campo psicoanalítico también hay una gran cantidad de propuestas psicoterapéuticas sintomales. Los tratamientos breves procuran un trabajo focalizado (Braier, 1984), sintomal. Según Atxotegi (1994, p. 36), las psicoterapias ${ }^{5}$ surgieron por dos motivos: la demanda creciente de asistencia psicológica en diversas instituciones; y la "necesidad de adaptar la técnica psicoanalítica a toda la gama de trastornos psicopatológicos y no tan sólo a los trastornos de tipo neurótico abordados por la técnica clásica". Para esta autora existe la cura-tipo o tratamiento psicoanalítico clásico; y, además, las psicoterapias psicoanalíticas, que tienen objetivos limitados. Entre estas últimas, se refiere a psicoterapia psicoanalítica (véase también Bolívar, 1990), psicoterapia breve o focal, psicoterapia de apoyo, psicoterapia institucional, etc. Por nuestra parte incluimos la consultoría psicoanalítica en las propuestas sintomales.

La focalización terapéutica puede definirse como la restricción del trabajo a un aspecto de la vida del paciente (Ramírez, 2012b). Esta manera de proceder ha sido objeto de variadas críticas, en particular, por parte de quienes consideran que el tratamiento psicoanalítico clásico es el único válido. No obstante, muchas circunstancias de la vida requieren este tipo de intervención (un sufrimiento intolerable derivado de un síntoma crónico, el deseo del paciente de trabajar un solo aspecto de su vida, restricciones institucionales, limitaciones de tiempo y dinero, formación y deseo del terapeuta). La focalización puede tener efectos insospechados, pues constituye una acción local, puntual que, en muchos casos, es la vía más idónea para iniciar cambios en otros niveles. Es frecuente que grandes cambios se hayan posibilitado a partir de modificaciones que parecían insignificantes en principio. Desde esta óptica no habría oposición entre la vertiente ascética y la vertiente sintomal: la primera puede

5 Aquí la autora se refiere con psicoterapias psicoanalíticas a todos aquellos tratamientos que derivan del psicoanálisis y que se diferencian claramente de éste (Atxotegi, 1994). En nuestro caso, hemos considerado el tratamiento psicoanalítico como una psicoterapia ascética; por ello, no plantearíamos una diferencia tajante entre psicoterapia y psicoanálisis. Esta es una de las razones por las cuales hemos preferido establecer las categorías de ascéticas y sintomales (Lopera, 2006). 
Desde nuestro punto de vista, la categorización de las psicoterapias en ascéticas y sintomales permite pensar aspectos comunes sin desconocer las diferencias. Consideramos mucho más productivo este abordaje que aquel que solo resalta las discordancias. Además, posibilita ver que hay una gama de propuestas que va desde las que intervienen en aspectos puntuales y focalizados de la vida de las personas, hasta aquellos que abarcan su existencia, su historia, sus vicisitudes, de la manera más amplia posible. producir cambios puntuales y la segunda puede iniciar un proceso de mayor alcance que el imaginado inicialmente.

Desde nuestro punto de vista, la categorización de las psicoterapias en ascéticas y sintomales permite pensar aspectos comunes sin desconocer las diferencias. Consideramos mucho más productivo este abordaje que aquel que solo resalta las discordancias. Además, posibilita ver que hay una gama de propuestas que va desde las que intervienen en aspectos puntuales y focalizados de la vida de las personas, hasta aquellos que abarcan su existencia, su historia, sus vicisitudes, de la manera más amplia posible. Así tenemos: la consultoría psicoanalítica, la terapia breve de orientación psicoanalítica, la psicoterapia psicoanalítica, el análisis terapéutico, la cura psicoanalítica (Ramírez, et al, 2015). Otto Kernberg (2001) diferencia entre psicoanálisis, psicoterapia psicoanalítica y psicoterapia de reforzamiento, todas basadas en los presupuestos conceptuales psicoanalíticos. Estas tres modalidades de tratamiento coinciden, a su juicio, en el uso de la interpretación, el manejo de la transferencia y la neutralidad técnica; ahora bien, difieren en el uso específico que hacen de cada una de estas tres estrategias técnicas: el psicoanálisis las emplea con el mayor rigor posible, en el sentido clásico freudiano; la psicoterapia psicoanalítica introduce algunas variaciones, como por ejemplo, tener más en cuenta el aquí y el ahora al momento de interpretar y manejar la transferencia; y la psicoterapia de reforzamiento acude más a la actitud directiva del terapeuta (conserva menos la neutralidad técnica) y se mezcla más con la sugestión (Kernberg, 2001).

Ahora bien, esta categorización se articula con otra, que también establece diferencias y afinidades entre diversos modelos. Nos referimos a tres enfoques de trabajo que hemos denominado: a) psicoterapias que ofrecen conocimientos psicológicos al consultante, b) psicoterapias que se centran en la relación terapéutica y c) psicoterapias que transmiten una actitud al paciente; las cuales desarrollamos a continuación.

\section{Transmisión del conocimiento psicológico}

La transmisión de teoría al paciente - recomendarle lecturas específicas sobre técnicas o sobre la concepción psicológica en la que se sustenta el modelo psicoterapéutico- está prácticamente ausente de las psicoterapias psicoanalíticas. Es más acorde con los modelos conductuales y cognitivos clásicos (Caro, 1997). Sin embargo, el lugar que ocupa la teoría en el proceder del clínico psicoanalítico es aún asunto de debate. Muchos sostienen que la teoría debe ser una guía y una perspectiva para leer e interpretar las manifestaciones y expresiones del paciente. Freud en ocasiones consideró importante el papel de la teoría para la comprensión de un caso. No obstante, en la descripción que hace de la técnica de la atención flotante, muestra con claridad que el analista debe escuchar suspendiendo en lo posible sus propias expectativas y sus concepciones teóricas previas, pues de lo contrario, nunca hallará nada nuevo (Freud, 1912/1998, pp. 111-112).

En las psicoterapias psicoanalíticas la teoría es entendida desde sus relaciones con la experiencia, esto es, con el proceso psicoterapéutico vivido por el mismo psicoterapeuta, en su proceso de formación. En primer lugar, porque dicha teoría deriva de la experiencia acumulada de los psicoterapeutas; y en segundo lugar, porque se considera que puede ser comprendida cuando ha sido incorporada por el clínico, es decir, cuando ha sido vivida.

Las diferencias en las concepciones teóricas llevan a distintas intervenciones. Desde la teoría de las relaciones objetales se concibe lo psíquico como resultado del 
encuentro entre la tendencia innata a la relación y el ambiente materno que le corresponde. Se rechaza la teoría clásica de que lo psíquico está regido por el principio de placer-displacer y que la pulsión sería esencial en su proceso de estructuración. Se relativiza - y en algunos casos se rechaza- el concepto de pulsión de muerte, y se da mayor importancia a la tendencia humana a la realización y despliegue vital.

A partir de estas diferencias teóricas se procede en el trabajo psicoterapéutico: ya sea otorgando mayor importancia a la calidad de la relación que se establece entre el analista y el analizando, o privilegiando el lugar del analista como un objeto pulsional al que se dirigiría la libido del paciente en el proceso transferencial. También hay diferencias en la concepción de la sexualidad, el cuerpo y la agresión, como ocurre entre Kohut y Kernberg, derivando en intervenciones distintas (Mitchel \& Black, 2004, p. 285). Para Kohut, "El analista sagaz, en especial si conoce la teoría de la psicología del sí-mismo, verá en la mentira de su paciente (al menos en ciertos casos) una primera, tímida afirmación de sus derechos como individualidad independiente (...)" (1993, p.117).

[El analista] (...) resistirá la tentación de amoldar su entendimiento del paciente a preconceptos teóricos rígidos -ya procedan de la teoría de Klein, de Rank, de Jung, de Adler, del análisis clásico, o incluso ¿por qué no?, de la psicología del sí mismo-, hasta que haya podido captar con más precisión la necesidad esencial del paciente y transmitirle ese entendimiento mediante una interpretación más correcta (Kohut, 1993, pp.108-109).

Kernberg (1993) destaca la confluencia de los diferentes modelos en una actitud que llama indoctrinación. Para él, es evidente la amplia preocupación respecto a los riesgos de seducir involuntariamente al analizando a la teoría o al lenguaje del analista.

\section{La relación terapéutica}

En esta categoría o enfoque de trabajo la teoría se ubica en un lugar secundario. Desde muy temprano Freud se percató de la importancia de la relación del paciente con el analista, a la que llamó transferencia. Consideró que un tratamiento psicoanalítico requería la instalación de una neurosis artificial o de transferencia y su resolución definitiva a través de su análisis (Freud, 1917/1998). La concepción sobre lo psíquico, así como su propia experiencia clínica, le mostraron que la actitud del analista debía ser la de abstinencia con respecto a expresar sus sentimientos y afectos hacia el paciente.

Otros teóricos, por el contrario, consideran que el analista debe ser cálido y afectuoso en la relación establecida con su paciente, procurando recrear el ambiente suficientemente bueno que es en sí mismo reparador. Para Winnicott (1998) la relación terapéutica no es la que cura en sí misma, aunque, por supuesto, no se le resta importancia. En la relación terapéutica el protagonista es el paciente, y el analista sólo ofrece el medio especializado para la curación, así como una técnica adecuada para tal propósito. Según Fairbairn (1962) lo que posibilita la cura es la relación del paciente con el analista, más allá de los detalles de la técnica. Para Guntrip, el supuesto fundamental sobre el cual descansa el tratamiento psicoanalítico es el establecimiento de una relación confiable entre el analista y el paciente, relación que estimula la capacidad de insight de este último y que puede ser terapéutica (Guntrip, 1971, p. 175). La psicoterapia no puede ser concebida como una técnica, "sino tan solo como la posibilidad de una relación personal genuina, capaz de brindar confianza, comprensión y respeto" (Guntrip, 1971, p. 180). Kohut señala que una buena 
En la orientación psicoanalítica en la psicoterapia (Lopera, 2004) se busca la moderación del sufrimiento a través de la transmisión de una actitud de escucha, análisis y respuesta. relación entre el analista y el paciente permite a este último vivir una experiencia de resonancia empática que se constituye en fundamento de su sentimiento de seguridad (1993, p. 114).

Desde modelos humanistas (Rogers, 1972), existenciales (Binswanger. 1973, p. 122) y fenomenológicos (Orange, 2013, 2012), se ha dado también un lugar central a los efectos terapéuticos de dicha relación; incluso en los modelos cognitivos posracionalistas (Mahoney \& Oyler, 1997).

\section{La transmisión de una actitud}

En esta categoría se parte del supuesto de que el paciente es quien debe tomar sus propias decisiones y que el psicoterapeuta es sólo un medio para ello. Se procura que la propia teoría sea puesta en suspenso o dejada a un lado mientras se escucha al paciente, y que lo que guíe el trabajo psicoterapéutico sea más bien un método, una actitud, que se expresa como docta ignorancia fundamentada en la aceptación de la propia ignorancia sobre la realidad del otro y en el reconocimiento del saber sobre un método de análisis, de un camino que permite a cada paciente acceder a su propia verdad (Lopera, Ramírez et al., 2010).

Esta actitud varía de acuerdo con cada enfoque de trabajo: quienes hacen énfasis en el establecimiento de una confianza básica, enseñan al paciente la importancia de ese tipo de vínculo. Quienes sostienen la conveniencia de analizar los problemas pensarlos, reflexionarlos, resignificarlos - transmiten al paciente la importancia de analizar. No se transmite entonces una teoría abstracta sobre lo psíquico, sino más bien una actitud, una forma de ver la vida. Para Freud se trata de permitir al paciente enfrentarse a su verdad, para que tome sus propias decisiones y elija su estilo de vida (Freud, 1917/1998, p. 395). Cuando Guntrip (1971) concibe la psicoterapia como una experiencia de relación genuina que posibilita confianza, comprensión y respeto, eso es precisamente lo que muestra y transmite a sus pacientes como lo más valioso de su trabajo. En rigor no es una teoría sino una actitud. Lacan propone, como esencial, la transmisión del método analítico, pues afirma: "Si tan sólo les hubiese enseñado aquí este método implacable de comentario de significantes, esto no habría sido en vano, al menos así lo espero. Espero incluso que no les quede nada más" (Lacan, 1988, p. 303).

En la orientación psicoanalítica en la psicoterapia (Lopera, 2004) se busca la moderación del sufrimiento a través de la transmisión de una actitud de escucha, análisis y respuesta. Lo que se transmite en este caso es el método analítico; al respecto Ramírez expresa:

No se trata entonces de enseñarle, y mucho menos imponerle una forma diferente, "mejor" de vivir. Él mismo, al reconocer su deseo, ha de responsabilizarse por su destino y decidir el camino que habrá de recorrer, las renuncias, aceptaciones y modificaciones que elegirá (Ramírez, 2012a, p. 367).

Para Schnitter (2004), el trabajo psicoterapéutico, como una de las modalidades del trabajo clínico, sería más un modelo de investigación, es decir, una forma de razonamiento que se fundamenta en la relación establecida entre el clínico y su paciente. Afirma:

Podemos definir la clínica como método de investigación, como una forma de razonamiento sobre cierto tipo de datos. Esta idea no es nueva. En el trabajo 
Pág 93

Las conceptualizaciones sobre lo psíquico -estructura, desarrollo y expresionestienen una incidencia directa en la manera como se lleva a cabo el trabajo psicoterapéutico. de Piaget sobre el pensamiento esto es evidente y en la obra Freudiana podemos ver el ejemplo de la diversidad de la clínica como método de investigación y como forma de intervención (2004, p. 6).

\section{Conclusiones}

La psicoterapia tiene una larga tradición filosófica si la entendemos como cuidado y cultivo de sí, de la propia alma. La expresión therapeuein heauton (cuidado de sí) (Foucault, 2002, p. 110; 1987) muestra cómo la cura de sí, tan importante en la filosofía antigua, era considerada esencial en la definición misma de la filosofía como manera de vivir (Hadot, 1998; 2006; 2009).

La clasificación en psicoterapias ascéticas y sintomales permite un examen más riguroso de cada propuesta, y más acorde con sus criterios. Muchas de las críticas a las psicoterapias psicoanalíticas (que pretenden una ascesis subjetiva) se basan en criterios que son exclusivamente aplicables a las psicoterapias sintomales, lo cual es un error; asimismo, los cuestionamientos realizados a las psicoterapias breves el que se les conciba como la aplicación del cobre de la sugestión (Freud, 1919/1998, p. 163) ${ }^{6}$ y se piense que siempre sería mejor una trabajo diferente- desconocen que precisamente sus objetivos buscan una eficacia sintomal, para lo cual utilizan la focalización como estrategia fundamental.

La focalización, que apunta a un aspecto concreto, no se opone a un trabajo que pretenda la revisión exhaustiva de la existencia de un paciente. Cambios puntuales y en apariencia triviales pueden generar o, al menos promover, un proceso de movimiento psíquico cuyas consecuencias últimas se desconocen, aunque quizá tiendan a un cuidado de sí. Incluso, en muchas ocasiones las pequeñas modificaciones son las únicas posibles dadas las circunstancias.

Las conceptualizaciones sobre lo psíquico -estructura, desarrollo y expresionestienen una incidencia directa en la manera como se lleva a cabo el trabajo psicoterapéutico. Sin embargo, estas teorías hacen parte de la experiencia acumulada de los terapeutas y son tomadas de manera flexible, al punto de que se da más énfasis a otros aspectos como, por ejemplo, a la relación terapéutica o a la transmisión de una actitud. Esto permite comprender por qué autores como Schnitter hablen de clínica psicológica y no de psicología clínica, puesto que, a su modo de ver, no se trata de la aplicación de la psicología general al ámbito clínico - o al psicoterapéutico-, sino más bien, de un modo de razonamiento que consiste en un emergente no establecido de antemano: "(...) el método es un emergente, se va construyendo en el contexto de la relación entre los sujetos" (2004, p. 5). Esta concepción de la clínica es completamente coincidente con la que desarrolla Manrique et al. (2016).

Si se pone en cuestión la idea clásica de que la psicología clínica es la aplicación de los conocimientos psicológicos generales y, además, se privilegia la transmisión de una actitud - no de la teoría preestablecida-, entonces la psicoterapia, como una modalidad de aplicación de la clínica psicológica, no sería una ciencia aplicada como proponen algunos modelos conductuales y cognitivos clásicos. Se trataría de la aplicación de un método o de una actitud científicos. De allí que se hable de modo de razonamiento, método analítico (Lopera, Ramírez et al., 2010), o establecimiento de una actitud de confianza básica que permita las condiciones más óptimas para la relación.

6 En este aspecto diferimos de algunos planteamientos de Freud que, con esta afirmación, negaba otras posibilidades del método psicoanalítico, diferentes a las de la cura-tipo, o análisis terapéutico. Paradójicamente, él mismo había desarrollado estas posibilidades en otras obras. Cfr. Sus libros Interpretación de los sueños, Psicopatología de la vida cotidiana, El chiste y su relación con lo inconsciente, entre otras (Freud, 1900/1998; 1901/1998; 1905/1988). 
Esta forma de concebir el proceso psicoterapéutico - como un emergente de la relación entre el psicoterapeuta y el paciente-reconoce la importancia del azar, la incertidumbre y el devenir en los sistemas complejos no simulables (Pagels, 1991). El ser humano es precisamente una de estas realidades complejas, y su abordaje psicoterapéutico ha de tener en cuenta estas características en lugar de ignorarlas. Al respecto, Schnitter plantea que: "[...] el razonamiento clínico debe incluir la diversidad, la incertidumbre y el movimiento, no la exactitud de la clasificación, sino el contexto vincular o relacional con el sujeto" (2004, p. 9).

El intento de establecer confluencias - sin desconocer las divergencias- entre los diferentes modelos a partir de la clasificación en ascéticos y sintomales, por un lado, y de los énfasis dados al conocimiento psicológico, a la relación terapéutica y a la transmisión de una actitud, por el otro, pretende resaltar una perspectiva indogmática y pluralista, desde la cual es posible concebir el propio modelo psicoterapéutico como uno más entre otros, y trascender así las posiciones radicales que proceden descalificando las demás propuestas y sólo considerando como válidas las propias. No significa renunciar a la psicoterapia que es de nuestra preferencia; se trata de abrirse a otros discursos, dejarse permear y enriquecerse de los aportes de los demás, creando así una verdadera comunidad de trabajo y de discusión.

\section{Referencias}

Aubenque, P. (1999). La prudencia en Aristóteles. Barcelona: Grijalbo Mondadori.

Atxotegi, J. (1994). "Delimitación conceptual del área de las psicoterapias psicoanalíticas". En A. Ávila. Manual de técnicas de psicoterapia. Un enfoque psicoanalítico. Madrid: Siglo XXI.

Aristóteles. (1985). Ética nicomáquea. Ética eudemia. Madrid: Gredos.

Binswanger, L. (1973). Artículos y conferencias escogidas. Madrid: Gredos.

Bleichmar, N., \& Leiberman, C. (1997). El psicoanálisis después de Freud. México: Paidós.

Brett, G. (1972). Historia de la psicología. Buenos Aires: Paidós.

Bolívar, G. (1990). Hacia un modelo de psicoterapia con orientación psicoanalítica. Monografía de psicología. Medellín: Departamento de Psicología, Universidad de Antioquia.

Braier, E. (1984). Psicoterapia breve de orientación psicoanalítica. Buenos Aires: Nueva Visión.

Caro, I. (1997). Manual de psicoterapias cognitivas. Barcelona: Paidós.

Cassirer, E. (1994 [1932]). Filosofía de la llustración. México: Fondo de Cultura Económica.

Consejo Directivo Universidad de Antioquia. (1975). Resolución 89 del 5 de agosto de 1975. Medellín: Universidad de Antioquia.

Consejo Directivo Universidad de Antioquia. (1979). Acuerdo N. 12 de 1979, junio 25. Medellín: Universidad de Antioquia.

Consejo Superior Universidad de Antioquia. (1991). Acuerdo Superior 196 de 19 de noviembre de 1991, "Por el cual se crea el Departamento de Psicoanálisis, adscrito a la Facultad de Ciencias Sociales y Humanas". Medellín: Universidad de Antioquia.

Departamento de Psicología Universidad de Antioquia. (1986). Reestructuración del Programa de Psicología. Octubre 31 de 1986. Medellín: Departamento de Psicología, Facultad de Ciencias Sociales, Universidad de Antioquia.

Departamento de Psicología Universidad de Antioquia. (2008). Proyecto Educativo del Programa (PEP). Medellín: Departamento de Psicología, Facultad de Ciencias Sociales y Humanas, Universidad de Antioquia. 
Descartes, R. (2008/1637). Discurso del método y Meditaciones metafísicas. Madrid: Tecnos.

Dilthey, W. (1945). Psicología y teoría del conocimiento. México: Fondo de Cultura Económica.

Eysenck, H., \& Wilson, G. (1980). El estudio experimental de las teorías freudianas. Madrid: Alianza Editorial.

Fairbairn, R. (1962). Estudio psicoanalítico de la personalidad. Buenos Aires: Hormé.

Foucault, M. (2002). Hermenéutica del sujeto. México: Fondo de Cultura Económica.

Foucault, M. (2000). "La ética del cuidado de sí como práctica de la libertad". Nombres. Revista de Filosofía, 10(5), 257-280.

Foucault, M. (1993). Historia de la sexualidad. 2. El uso de los placeres. México: Siglo $X X I$.

Foucault, M. (1991). Vigilar y castigar. México: Siglo XXI.

Foucault, M. (1990). Tecnologías del yo. Barcelona: Paidós.

Foucault, M. (1987). Historia de la sexualidad. 3. La inquietud de sí. México: Siglo XXI.

Freud, S. (1998). Tratamiento psíquico (tratamiento del alma). En J. L. Etcheverry (Traduc.). Obras completas: Sigmund Freud (Vol. 1, pp. 11-132). Buenos Aires: Amorrortu. (Trabajo original publicado en 1890).

Freud, S. (1998). Estudios sobre la histeria. En J. L. Etcheverry (Traduc.). Obras completas: Sigmund Freud (Vol. 2). Buenos Aires: Amorrortu. (Trabajo original publicado en 1895).

Freud, S. (1998). Interpretación de los sueños. En J. L. Etcheverry (Traduc.). Obras completas: Sigmund Freud (Vol. 5). Buenos Aires: Amorrortu. (Trabajo original publicado en 1895).

Freud, S. (1998). Psicopatología de la vida cotidiana. En J. L. Etcheverry (Traduc.). Obras completas: Sigmund Freud (Vol. 6). Buenos Aires: Amorrortu. (Trabajo original publicado en 1901).

Freud, S. (1998). El chiste y su relación con lo inconciente. En J. L. Etcheverry (Traduc.). Obras completas: Sigmund Freud (Vol. 8). Buenos Aires: Amorrortu. (Trabajo original publicado en 1905).

Freud, S. (1998). Consejos al médico sobre el tratamiento psicoanalítico y Sobre la iniciación del tratamiento. En J. L. Etcheverry (Traduc.). Obras completas: Sigmund Freud (Vol. 12, pp. 107-120). Buenos Aires: Amorrortu. (Trabajo original publicado en 1912).

Freud, S. (1998). La represión. En J. L. Etcheverry (Traduc.). Obras completas: Sigmund Freud (Vol. 14, pp. 135-152). Buenos Aires: Amorrortu. (Trabajo original publicado en 1915).

Freud, S. (1998). Introducción al psicoanálisis. $27^{\text {a }}$ Conferencia. La transferencia. En J. L. Etcheverry (Traduc.). Obras completas: Sigmund Freud (Vol. 16, pp. 392-407). Buenos Aires: Amorrortu. (Trabajo original publicado en 1917).

Freud, S. (1998). Nuevos caminos de la terapia psicoanalítica. En J. L. Etcheverry (Traduc.). Obras completas: Sigmund Freud (Vol. 17, pp. 151-154). Buenos Aires: Amorrortu. (Trabajo original publicado en 1919).

Freud, S. (1998). Dos artículos para enciclopedia: «psicoanálisis» y «teoría de la libido». En J. L. Etcheverry (Traduc.). Obras completas: Sigmund Freud (Vol. 18, pp. 227-254). Buenos Aires: Amorrortu (Trabajo original publicado en 1923).

Freud, S. (1998) ¿Pueden los legos ejercer el análisis? Diálogos con un juez imparcial. En J. L. Etcheverry (Traduc.). Obras completas: Sigmund Freud (Vol. 20, pp. 165-242). Buenos Aires: Amorrortu. Trabajo original publicado en 1926).

Freud, S. (1998). El porvenir de una ilusión. En J. L. Etcheverry (Traduc.). Obras completas: Sigmund Freud (Vol. 21, pp. 1-56). Buenos Aires: Amorrortu. (Trabajo original publicado en 1927). 
Gil, L.M. (1995). El método analítico en la práctica psicológica. Monografía de Psicología. Medellín: Departamento de Psicología, Universidad de Antioquia.

Ginzburg, C. (1989). Morelli, Freud y Sherlock Holmes: indicios y método científico. En U. Eco, \& T. Sebeok (Comp.). (1989) El signo de los tres. Barcelona: Lumen.

Guntrip, H. (1971). El "self" en la teoría y en la terapia psicoanalíticas. Buenos Aires: Amorrortu.

Hadot. P. (1998). ¿Qué es la filosofía antigua? México: Fondo de Cultura Económica.

Hadot. P. (2006). Ejercicios espirituales y filosofía antigua. Madrid: Ediciones Siruela.

Hadot, P. (2009). La filosofía como forma de vida. Conversaciones con Jeannie Carlier y Arnold I. Davidson. Barcelona: Alpha Decay.

Hartmann, H. (1964). Ensayos sobre la psicología del yo. México: Fondo de Cultura Económica.

Instituto Colombiano para el Fomento de la Educación Superior (ICFES). (1975). Acuerdo 209 del 12 de septiembre de 1975: "Por el cual se aplaza la decisión acerca de la Licencia de Iniciación de Labores para el Programa de Psicología de la Universidad de Antioquia". Bogotá: ICFES.

Instituto Colombiano para el Fomento de la Educación Superior (ICFES). (1977). Acuerdo Número 88 de 1977: "Por el cual se concede Licencia de Iniciación de Labores al Programa de Psicología de la Universidad de Antioquia". Bogotá: ICFES.

Instituto Colombiano para el Fomento de la Educación Superior (ICFES). (1982). Acuerdo 199 de diciembre 16 de 1982. "Por el cual se concede Licencia de Funcionamiento al Programa de Psicología dela Universidad de Antioquia, con sede en Medellín". Bogotá: ICFES.

Instituto Colombiano para el Fomento de la Educación Superior (ICFES). (1984). Resolución 1577 de 1984. Bogotá: ICFES.

Jardim, L., \& Rojas, M. (2010). "Investigación psicoanalítica en la universidad”. Estudos de Psicologia, 27(4), 529-536.

Kernberg, 0. (2001). "Psicoanálisis, psicoterapia psicoanalítica y psicoterapia de fortalecimiento: controversias contemporáneas". Psicoanálisis APdeBA, 23(1), 201 227.

Kernberg, 0. (1993). Convergencias y divergencias en la técnica psicoanalítica contemporánea. Libro Anual de la IPA, N IX, 129-143. Londres: Institute of Psychcoanalysis,

Kohut, H. (1993). Cómo cura el análisis. Buenos Aires: Paidós.

Lacan, J. (1983). Seminario 2. El yo en la teoría de Freud y en la técnica psicoanalítica. Barcelona: Paidós.

Lacan, J. (1988). Seminario 7. La ética del psicoanálisis. Barcelona: Paidós.

León, S. (s.f.). "El lugar del psicoanálisis en la Universidad". Recuperado de: http:// www.facso.uchile.cl/psicologia/caps/pdf/pn universidad.pdf

Lopera, J. (2016). Sabiduría práctica y salud psíquica. Bogotá: Editorial San Pablo y Fondo Editorial EAFIT.

Lopera, J. (2015). "A propósito del programa de psicología de la Universidad de Antioquia en sus 40 años". Revista de Psicología Universidad de Antioquia, 7(2), 61-176.

Lopera, J. (2006). "Psicología ascética y psicología epistémica". Acta Colombiana de Psicología, 9(2), 75-86.

Lopera, J. (2004). La orientación psicoanalítica en la psicoterapia. Memorias Primer Encuentro Nacional de Psicoterapias Dinámicas. Universidad de Antioquia.

Lopera, J., Ramírez, C., Zuluaga, M., \& Ortiz, J. (2010). El método analítico. Medellín: Centro de Investigaciones Sociales y Humanas (CISH) de la Universidad de Antioquia. 
Lopera, J., Manrique, H., Zuluaga M., \& Ortiz, J. (2010). El objeto de la psicología: el alma como cultura encarnada. Medellín: Editorial Universidad de Antioquia.

López, R. (1995). Momentos del psicoanálisis en Colombia. Medellín: Editorial El Propio Bolsillo.

Mahoney, M., \& Oyler, J. (1997). El proceso terapéutico en la psicoterapia constructiva. En Isabel Caro (Comp.). Manual de Psicoterapias cognitivas. Barcelona: Paidós.

Mahler, M. (1972). Simbiosis humana. Las vicisitudes de la individualización. Medellín: Joaquín Mortiz.

Manrique, H., Lopera, I., Pérez, J., Ramírez, V., \& Henao, C. (2016). Clínica analítica de las organizaciones. Bogotá: Editorial San Pablo y Fondo Editorial EAFIT.

Miller, J-A. (2001). "Psicoanálisis puro, psicoanálisis aplicado y psicoterapia". Recuperado de: https://elp.org.es/wp-content/uploads/2013/03/4 psicoa puro miller.pdf

Mitchel, S., \& Black, M. (2004). Más allá de Freud. Una historia del pensamiento psicoanalítico moderno. Barcelona: Herder.

Orange, D. (2013). El desconocido que sufre. Santiago de Chile: Editorial Cuatro Vientos.

Orange, D. (2012). Pensar la práctica clínica. Recursos filosóficos para el psicoanálisis y las psicoterapias humanistas. Santiago de Chile: Editorial Cuatro Vientos.

Pagels, H. (1991). Los sueños de la razón: el ordenador y los nuevos horizontes de las ciencias de la complejidad. Barcelona: Gedisa.

Pérez, J. (1992). "Psicología y psicoanálisis". Revista Colombiana de Psicología, (1), 54-58.

Platón. (1985). "Protágoras". En Diálogos I. Madrid: Gredos.

Platón. (1988). "Fedón”. En Diálogos III. Madrid: Gredos.

Ramírez, C. (1991). El método científico en el psicoanálisis. Revista Universidad de Antioquia, LX(224), 35-41.

Ramírez, C. (2011). "Orientación psicoanalítica en la psicología”. Artículo 19. Apuntes. Medellín: Grupo de investigación El método analítico y sus aplicaciones en las ciencias sociales y humanas.

Ramírez, C. (2012a) "Psicopatología". Ens. 240. En La vida como un juego existencial: ensayitos. Medellín: Fondo Editorial EAFIT.

Ramírez, C. (2012b) "Focalización". Ens. 269. En La vida como un juego existencial: ensayitos. Medellín: Fondo Editorial EAFIT.

Ramírez, C., Lopera, J., Zuluaga, M., Henao, C., Ramírez, V., \& Carmona, D., (2015). Relaciones psicología-psicoanálisis. Bogotá: San Pablo y Fondo Editorial EAFIT.

Ramírez, M. (1994). “Psicología psicoanalítica?”. Psique, Revista de Psicología, (5), 55-67.

Rogers, C. (1972). Psicoterapia centrada en el cliente. (3.th. ed.) Buenos Aires: Paidós.

Santamaría, R. (2002). "Acerca del método psicoanalítico de investigación". En Universidades [en linea] Recuperado de: http://www.redalyc.org/articulo. oa? id $=37302307$

Sección de Servicios del Departamento de Psicología Universidad de Antioquia. (1989). Carta del 04 de agosto de 1989 al Consejo Académico de los profesores de la Sección de Servicios del Departamento de Psicología de la Facultad de Ciencias y Humanidades. Medellín: Universidad de Antioquia.

Schnitter, M. (2004). Psicoterapia Dinámica: La Perspectiva Desde La Psicología Relacional. La Reinvención de la Clínica. [CD Room] Memorias Primer Encuentro Nacional de Psicoterapias Dinámicas. Medellín: Universidad de Antioquia.

Watson, J. (1982/1916). "La psicología tal como la ve el conductista”. En J., Gondra. La psicología moderna. Textos básicos para su génesis y desarrollo. Bilbao: Desclée de Brouwer. 
Winnicott, D. (1998). Escritos de pediatría y psicoanálisis. Barcelona: Paidós.

Wundt, W. (1982/1896). "Objeto, divisiones y método de la psicología". En J., Gondra. La psicología moderna. Textos básicos para su génesis y desarrollo. Bilbao: Desclée de Brouwer.

Wyss, D. (1964). Las escuelas de psicología profunda desde sus principios hasta la actualidad. Madrid: Gredos. 